\title{
RNA folding retrospective: lessons from ribozymes big and small
}

\author{
SARAH A. WOODSON \\ T. C. Jenkins Department of Biophysics, Johns Hopkins University, Baltimore, Maryland 21218, USA
}

The past 20 years have brought an explosion of structural and physical information about RNAs and their complexes. A vast network of non-coding RNA sequences are now believed to be essential for the complexity of life. It is now possible to interrogate RNA structures across an entire transcriptome, and recent experiments have turned up a dizzying array of RNA binding interactions, chemical modifications and small non-coding RNAs that fine-tune mRNA life-cycles. Yet, the need to understand and predict how RNAs take on particular structures is more urgent than ever before. This is not only to interpret the possible functions of newly discovered noncoding RNA sequences, but also to appreciate how dynamical motions and conformational switches drive catalysis and gene regulation. Of course, cellular machines must self-assemble from their components, and thus the principles of self-assembly also underlie the design of synthetic RNAs.

The discovery of catalytic RNA motivated fresh studies of RNA structure and assembly, which were beginning to accelerate around the start of the RNA journal in 1995. Nevertheless, the RNA folding story started with experiments on tRNA and small hairpins in the 1970s. These early experiments established nucleation-zippering models for basepairing and that neighboring base-pair interactions were sufficient to predict the stability of a double helix. Nearly all the current structure-prediction programs use nearest-neighbor energy parameters refined over the years by Doug Turner and his colleagues. Experiments on tRNA established the hierarchy of folding from Watson-Crick base pairs (2D) to non-Watson-Crick tertiary interactions (3D). They also showed the importance of $\mathrm{Mg}^{2+}$ ions for stabilizing RNA tertiary structure (starting the discussion on how ions actually do this), and demonstrated the propensity of RNAs to form metastable structures.

My own pre-occupation with the mechanism of RNA folding started backwards, when I was trying to understand why the autocatalytic Tetrahymena group I intron could not reverse splice back into its natural splice junction. Like many group I introns, the well-studied Tetrahymena intron inter-

Corresponding author: swoodson@jhu.edu

Article and publication date are at http://www.rnajournal.org/cgi/doi/ 10.1261/rna.051110.115. Freely available online through the RNA Open Access option. rupts the genes encoding rRNA. My postdoctoral advisor Tom Cech and I realized that refolding of the spliced ribosomal RNA helped drive the self-splicing reaction forward. Later, I found that eight segments of the pre-ribosomal RNA can base-pair in two mutually exclusive patterns. One secondary structure allows the catalytic core of the intron, or ribozyme, to form the active $3 \mathrm{D}$ structure, resulting in rapid self-splicing. The alternative secondary structure misfolds the catalytic core, blocking $5^{\prime}$ splice site recognition and normal splicing. The concept of regulation via mutually exclusive RNA secondary structures had been elegantly worked out by Charles Yanofsky for anti-termination of the trp operon. Thousands of bacterial riboswitches are now known to use this same principle for controlling the transcriptional elongation of downstream genes.

The observation that the function of a self-splicing intron could be so strongly influenced by its genetic context set us on the path to understanding how large RNAs fold. A key finding was that a fraction of the RNA population folded correctly in a few seconds, while other molecules became kinetically trapped in misfolded structures. Dave Thirumalai and I showed that one could use the energy landscape theories developed for protein folding to describe the partitioning of RNA molecules along different folding paths. Singlemolecule experiments have abundantly demonstrated the heterogeneity of RNA folding kinetics and begun to illuminate how conditions can divide RNAs into subpopulations with different structures and functional properties. Kinetic partitioning of the Tetrahymena pre-RNA into its active structure can be altered by single mutations, and accounts for its intracellular abundance when the unspliced transcript is unstable.

What further principles of RNA folding have been learned by the field in the past 20 years? First, double helices can assemble into tightly packed structures that may be recognized by RNA binding proteins and small molecules. Proper helix assembly aligns the peripheral regions of the RNA so that they can make additional long-range tertiary interactions. This mutual cooperation between core and peripheral

(c) 2015 Woodson This article, published in $R N A$, is available under a Creative Commons License (Attribution-NonCommercial 4.0 International), as described at http://creativecommons.org/licenses/by-nc/4.0/. 
tertiary interactions biases the folding intermediates toward the correct structure, and reinforces functional sites that are often inherently unstable. The interplay between secondary structure, active sites and stabilizing tertiary interaction motifs can be read in the conservation and diversification of RNA sequence families over the course of molecular evolution.

Second, metals or other cations are needed to overcome the charge repulsion of the phosphate groups on the RNA backbone. Though this problem is far from solved, increasingly sophisticated experiments and theoretical simulations are beginning to tease out how condensation of cations around the RNA is coupled to assembly of compact tertiary structures. Small $\mathrm{Mg}^{2+}$ ions are most efficient, but $\mathrm{K}^{+}$and polyamines can also stabilize RNA tertiary structures, and likely do so in the cell. Interestingly, larger ions produce swollen structures that appear more loosely packed-a phenomena that will require more experiments and simulations to understand. High-resolution crystal structures and biochemical experiments have also shown that RNA active sites chelate dehydrated $\mathrm{Mg}^{2+}$ ions, which participate in catalysis. Neutral solutes that crowd the solution also stabilize RNA structures, perhaps explaining why many RNAs require less $\mathrm{Mg}^{2+}$ to act in the cell than in solution.

Third, RNAs are not still — they move, and these motions are thought to be important for substrate recognition, assembly of RNA-protein complexes (RNPs), and regulatory switches. Internal loops and helix junctions serve as hinges for large scale motions between helices, which are directional and can be captured by small ligands (drugs) or RNA binding proteins. Such motions are likely to be essential for the assembly of large RNPs, which nearly always require refolding of the RNA, and often refolding of the proteins as well. This process is best understood from stable RNPs such as the ribosome or signal recognition particle that are formed by the sequential addition of proteins, each of which changes the structure of the RNA in the complex. Nevertheless, there is still much to learn about how thermal motions are coupled to assembly, and how the dynamical properties of RNA-pro- tein interactions contribute to RNA processing or translational control.

Fourth, many non-coding RNA structures contain base pairs between nucleotides that are very far apart in the primary sequence. As a result, the $5^{\prime}$ end of the transcript must fold into a metastable intermediate structure, while waiting for the $3^{\prime}$ pairing partner to be transcribed. The coupling between the folding of nascent transcripts and the rate of transcription elongation has been elegantly demonstrated for several riboswitches. One common finding is that the location and duration of transcriptional pause sites can determine the folding pathway of the RNA and the probability of conformational switching in the presence of ligand. Many viruses also encode alternative metastable structures that control genomic replication and packaging.

Finally, the concepts outlined above have been worked out with many experiments and simulations in many laboratories. Increasingly sophisticated physical methods are capable of tracking motions in the RNA or distinguishing between subpopulations. These include single molecule fluorescence energy transfer (smFRET), optical tweezers, solution X-ray scattering, NMR, EPR, and cryo-electron microscopy. We have used time-resolved footprinting to capture RNA folding and RNP assembly in real time. Increasingly, such footprinting methods are being used to probe RNAs in living cells. Coupled with high-throughput sequencing methods, such strategies offer the exciting possibility of understanding how RNAs folding during transcription in the cell, or how they respond to changing patterns of RNA binding protein expression. This ability to capture RNA structures in situ is likely to be particularly important for getting a clear picture of long non-coding RNAs, that likely interact with RNA binding proteins during transcription. In the next 10 or 20 years of RNA folding, these and other methods will start to uncover the dynamic networks of RNA and protein interactions so important for gene regulation and adaptation. Nevertheless, as the RNA community gains a more sophisticated "systems" view of RNA structures, the principles learned from tRNAs will still be there to guide us. 

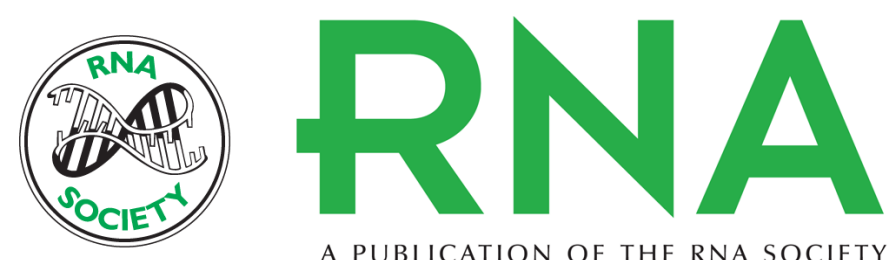

A PUBLICATION OF THE RNA SOCIETY

\title{
RNA folding retrospective: lessons from ribozymes big and small
}

\author{
Sarah A. Woodson
}

RNA 2015 21: 502-503
Open Access Freely available online through the RNA Open Access option.
Creative This article, published in $R N A$, is available under a Creative Commons License Commons (Attribution-NonCommercial 4.0 International), as described at License http://creativecommons.org/licenses/by-nc/4.0/.

Email Alerting Receive free email alerts when new articles cite this article - sign up in the box at the Service top right corner of the article or click here. 